SLEEP DISORDERED BREATHING

\title{
A simple procedure for measuring pharyngeal sensitivity: a contribution to the diagnosis of sleep apnoea
}

\author{
M Dematteis, P Lévy, J-L Pépin
}

Thorax 2005;60:418-426. doi: 10.1136/thx.2003.015032

See end of article for authors' affiliations

......................

Correspondence to: Dr M Dematteis, Laboratoire HP2 (HypoxiePhysioPathologies), Faculté de Médecine de Grenoble, Domaine de La Merci, 38706 La Tronche Cedex, France; maurice. dematteis@ujf-grenoble.fr

Received

11 September 2003

Accepted 31 October 2004
Background: Patients with severe apnoea may have an impaired pharyngeal dilating reflex related to decreased pharyngeal sensitivity. The accuracy of a simple new procedure to measure pharyngeal sensitivity and to diagnose sleep disordered breathing (SDB) was investigated.

Methods: Pharyngeal disappearance and appearance sensory perception thresholds were measured by delivering different airflow rates on the soft palate using an intraoral device in 17 controls and 50 patients suffering from SDB evaluated by overnight polygraphy. The procedure was performed before (baseline) and after three successive administrations of a topical anaesthetic to sensitise the pharyngeal sensory impairment. Pharyngeal sensitivity was then evaluated according to SDB severity. SDB was classified as mild, moderate or severe according to the relative proportion of obstructive apnoeas-hypopnoeas and the amount of desaturation.

Results: Patients had higher baseline disappearance and appearance sensory thresholds than controls (mean (SD) $0.62(0.44) \vee 0.26(0.06) \mathrm{l} / \mathrm{min}$ and $0.85(0.40) \vee 0.40(0.19) \mathrm{l} / \mathrm{min}, \mathrm{p}<0.001$, respectively). Such differences were enhanced by topical anaesthesia. Impairment of pharyngeal sensitivity and the number of patients with impaired sensitivity increased from the least to the most severe SDB group as indicated by the test sensitivity for a respiratory disturbance index of $>20 /$ hour $150 \%, 73.7 \%$ and $88.5 \%$ in the mild, moderate, and severely affected groups, respectively).

Conclusions: This simple and safe procedure showed that impairment of pharyngeal sensitivity is correlated with severity of SDB. Using this test in routine clinical practice may simplify the diagnosis of sleep apnoea, particularly for the most severe patients.
$\mathrm{S}$ leep disordered breathing (SDB) corresponds to a continuous clinical spectrum from snoring, upper airway resistance episodes, to obstructive hypopnoeas and apnoeas according to the severity of upper airway collapsibility. ${ }^{1}$ The common characteristic is a repetitive partial or complete collapse occurring during sleep at the pharyngeal level, a region lacking rigid support. ${ }^{2}$ Thus, pharyngeal patency is dependent on both its anatomy (calibre) and on the activity of pharyngeal dilator muscles (PDM) such as the genioglossus muscle. ${ }^{1}$ PDM have inspiratory phasic activity preceding diaphragmatic contraction, thus anticipating the development of intrapharyngeal negative pressure related to inspiration. ${ }^{3}$ This muscle activity is reduced during sleep leading to pharyngeal obstruction in patients with high pharyngeal collapsibility. ${ }^{4}$ There is increased evidence that upper airway mucosal sensory receptors may play a role in the patency of the upper airway through a reflex PDM activation. During wakefulness a negative pressure applied to the upper airway increases genioglossus muscle activity. ${ }^{5}$ This response is reduced by topical anaesthesia, suggesting that upper airway receptors may be involved in the afferent limb of this reflex. ${ }^{6}$ Anaesthesia of the upper airway increases pharyngeal airflow resistance, ${ }^{7}$ induces apnoeas/hypopnoeas in healthy subjects, ${ }^{8}$ and increases the frequency of obstructive events in snorers ${ }^{9}$ and the duration of apnoeas in apnoeic subjects. ${ }^{10}$ Thus, impairment of pharyngeal sensitivity may play a role in the pathophysiology of SDB through impairment of the pharyngeal dilator reflex.

Sleep apnoea syndrome is highly prevalent and represents a major public health problem. ${ }^{11}$ Diagnosis is by polysomnography which is expensive, labour intensive and time consuming. Simpler alternative diagnostic procedures are therefore strongly welcomed. Assessment of pharyngeal sensitivity could facilitate the diagnosis of SDB in some groups of patients with high clinical probability. While the anatomy of the upper airway can only predict the severity of SDB in young lean subjects who represent only a small proportion of SDB patients, ${ }^{12}$ evaluation of functional pharyngeal impairment may allow the presence and severity of SDB to be predicted.

We therefore investigated, in a prospective study, the accuracy of a new simple technique to evaluate whether impairment of pharyngeal sensitivity correlates with SDB severity and assessed the clinical usefulness of this procedure in the diagnosis of SDB.

\section{METHODS}

Study design

Seventeen controls and 50 patients with SDB (all men aged over 20 years who were not receiving any medication that may produce drowsiness and had no recognised cause of polyneuropathy, recent upper airway infection, or history of surgery of the upper airway except past tonsillectomy) were included in the study. Patients had SDB symptoms and a respiratory disturbance index (RDI) of $>20$ events/hour. Control subjects did not report any symptoms compatible with SDB such as habitual snoring, daytime fatigue or sleepiness, morning headache, and had normal nocturnal oximetry. In accordance with the ethical standards of the Grenoble University Hospital, all subjects gave informed consent to participate in the study.

Abbreviations: $\mathrm{AHI}$, apnoea/hypopnoea index; $\mathrm{BMI}$, body mass index; PDM, pharyngeal dilator muscles; RDI, respiratory disturbance index; $\mathrm{SDB}$, sleep disordered breathing 


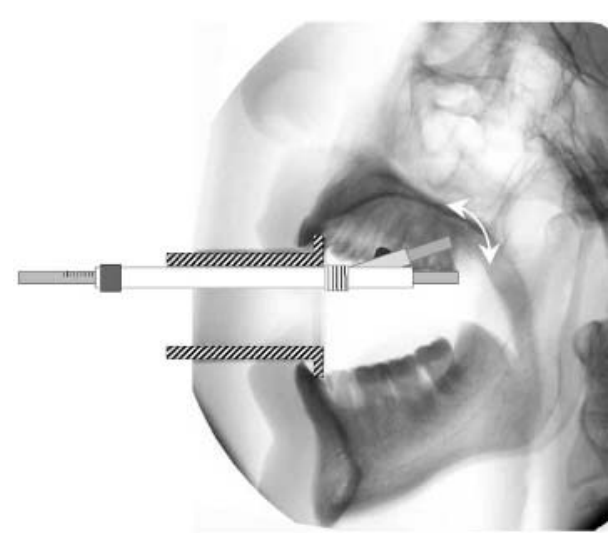

Figure 1 Device for the determination of pharyngeal sensory perception thresholds. Schematic representation of the device superimposed on a teleradiography. The device consists of an intraoral apparatus through which airflow is administered $1 \mathrm{~cm}$ in front of the central part of the soft palate mucosa using a graduated and adjustable catheter (white arrows).

\section{Pharyngeal sensory testing}

Apparatus and procedure

The apparatus consisted of an intraoral device through which airflow was applied on the soft palate using a catheter (fig 1). The catheter was graduated to allow length adjustments through a guide fixed into the upper part of a cylinder and articulated in its distal part.

Equipped with a headlamp, the investigator was able to control visually through the cylinder and to adjust the position of the catheter according to the anatomy of the tested subject to target the desired part of the pharynx. The cylinder had raised edges on the side introduced in the mouth, allowing the cylinder to be positioned with its edges against the posterior face of the incisors, a fixed anatomical landmark.

The pharyngeal sensory perception threshold was measured by varying airflow rates using the psychophysical method of limits. Sensory testing was most often performed the day after the diagnostic polygraphy or, at the latest, within the week following the sleep study and always before any SDB treatment. The test was conducted in a temperature controlled quiet room with only the subject and the investigator, blinded to the subject status for SDB. The subject wore a pair of earmuffs and was instructed to close his eyes to prevent the use of visual and auditory cues and to improve concentration. Wakefulness was maintained throughout the session test by regularly asking the subject to give his best response-that is, the smallest pharyngeal sensation that could be felt.

\section{Description of test session}

After description of the device and the procedure, the subject signed a consent form and characteristics were collected including clinical evaluation of the oropharyngeal cavity, scoring of gag reflex $(0=$ absent; $1=$ decreased; $2=$ normal; $3=$ exaggerated $)$ and spicy food consumption $(0=$ none; $1=$ little; 2 = moderate; $3=$ high consumption). The device was inserted into the subject's mouth and firmly maintained by the teeth and lips. The distal part of the guide was adjusted to aim at the central part of the soft palate. The catheter was introduced in the guide and gently pushed until contact with the mucosa, then pulled $1 \mathrm{~cm}$ back and fixed. The distance between the tip of the catheter and the mucosa was therefore similar for each tested subject, thus avoiding any difference in sensitivity due to catheter location. A detectable stimulus (suprathreshold stimulus, $2 \mathrm{l} / \mathrm{min}$ ) was administered before testing to familiarise the patient with the stimulus and to discuss the sensation with the investigator. The subject was instructed to report the smallest sensation felt. Appearance threshold was determined by progressively increasing the airflow (increments of $0.25 \mathrm{l} / \mathrm{min}$ between 0 and $2 \mathrm{l} / \mathrm{min}, 0.50 \mathrm{l} / \mathrm{min}$ between 2 and $3 \mathrm{l} / \mathrm{min}$, then $1 \mathrm{l} / \mathrm{min}$ above $3 \mathrm{l} / \mathrm{min}$ ) until the subject indicated the perception by raising his hand. The disappearance threshold was determined by progressively decreasing the suprathreshold airflow until the subject indicated its disappearance by lowering his hand. Each procedure was repeated at least twice and the values of at least three consecutive measurements were averaged.

To improve the sensitivity of the procedure, the test was repeated after successive administrations of a topical anaesthetic (Xylocaine 5\%, AstraZeneca Laboratory, France). We hypothesised that measuring pharyngeal sensitivity as previously described may not be sufficient to detect changes in sensitivity in patients suffering from mild neuropathy-that is, not severe enough to increase the baseline threshold perception. To avoid such limitation, pharyngeal sensitivity was therefore reassessed after graded mucosal anaesthesia. Application of an anaesthetic on diseased nerves should induce a greater anaesthesia (that is, higher sensation thresholds in patients with pharyngeal neuropathy) and allow better discrimination between patients and controls and between different subgroups of patients. One spray was applied to the test site and the procedure was repeated 5 minutes later, followed by measurements after two further applications of anaesthetic. Hence, for each subject, measurement of the appearance and disappearance of sensory detection thresholds at baseline and after successive administrations of topical anaesthetic enabled us to determine a slope between sensory thresholds obtained at baseline and after anaesthesia (appearance threshold slope and disappearance threshold slope, respectively), reflecting their response to anaesthesia. The slope was calculated using Excel software and corresponded to the slope of a linear regression for the measurements obtained at baseline and after anaesthesia.

\section{Quality control and repeatability of the procedure}

To prevent anticipation of the stimulus and distinction between external and internal stimuli-that is, the impression of feeling a sensation-null stimuli (similar procedure with no airflow) were randomly applied throughout sessions of the appearance threshold procedure. Signalling a perception during a null stimulus invalidated the test and the subject was re-instructed. To prevent interference between the airflow administered through the catheter and the breathing related airflow, the subject was instructed to breathe quietly through the nose.

The repeatability of the procedure (disappearance and appearance thresholds) was assessed only in control subjects since SDB treatment may improve the impairment of pharyngeal sensitivity. ${ }^{13}$ Ten controls were re-evaluated after a relatively long delay (mean (SD) 31.9 (3) weeks) to reduce possible learning effects.

\section{Sleep studies}

Overnight polygraphy included airflow assessment using nasal cannulae and a thermistor. Respiratory efforts were assessed using thoracic and abdominal movements and pulse transit time or by monitoring oesophageal pressure. Sleep stages were scored according to Rechtschaffen and Kales' criteria. Apnoea episodes were defined as complete airflow cessation for $>10$ seconds. Hypopnoeas were defined as a $50 \%$ decrease in airflow or a reduction in airflow of $30-50 \%$ with a microarousal or a $3 \%$ desaturation, both for 
$>10$ seconds. Inspiratory flow limitation episodes had a "plateau" aspect of the inspiratory flow curve of 10 seconds ending by a microarousal or returning to a rounded aspect of the flow curve. ${ }^{14}$ Apnoea and hypopnoea events were classified as obstructive based on the presence or the increase in respiratory effort, respectively. The RDI (number of apnoeas + hypopnoeas + flow limitation episodes) was considered abnormal above 20/hour of sleep.

\section{Statistical analysis}

For the 10 control subjects assessed twice, a two way analysis of variance (ANOVA) for repeated measurements was used to assess the effect of measurement conditions (baseline and successive anaesthesias) for each session, as well as the effect of session and the interaction between condition measurements and sessions. The repeatability of the procedure performed in these 10 control subjects was analysed in two different ways:

(1) Repeatability was first assessed using a one way ANOVA for repeated measurements. This analysis allowed estimation of the different components of variance required for the calculations of test-retest reliability-that is, the intraclass correlation coefficient (ICC 3,1). The ICC was defined by $($ BMS - EMS $) /($ BMS $+(n-1) \times$ EMS $)$ where BMS is the between subjects mean square and EMS is the error (residual) mean square. 95\% confidence intervals for the ICC values were calculated. The repeatability coefficient was calculated from the formula $1.96 \sqrt{ }(2 \times$ EMS $)$.

(2) Repeatability was further assessed using the BlandAltman procedure, plotting the differences between the repeated measurements ( $y$ axis) against their average (mean of the differences, $x$ axis). Since the Bland-Altman procedure assumes independent subjects, the calculations were done separately for measurements obtained at baseline and after each administration of anaesthetic for both the disappearance and appearance sensory thresholds. Using this method, $95 \%$ limits of agreement were calculated (mean $\pm 1.96 \mathrm{SD}-$ that is, the range in which the difference may be expected to lie in $95 \%$ of the measurements-where SD is the standard deviation of the differences between paired measurements); $95 \%$ confidence intervals (95\% CI) were calculated to indicate the precision of the limits of agreement. As a measure of repeatability, the British Standards Institution repeatability coefficient was calculated as 1.96 times the standard deviation of the differences. This coefficient indicates the maximum difference likely to occur between the measurements of the two sessions.

For the rest of the analyses, heterogeneity of variances (Levene's test) required the use of non-parametric tests to analyse the results, expressed as mean (SD) values. Intergroup comparisons were done with the Kruskall-Wallis test followed, if necessary, by a post hoc pairwise MannWhitney U test between controls and patients. The Spearman rank correlation test was used for correlation analysis between sensory values and anthropometric data and polysomnographic measurements, and was performed for the whole patient cohort. Pharyngeal sensitivity was then analysed according to SDB severity using classical indices such as apnoea/hypopnoea index (AHI) and RDI. However, since AHI and RDI only referred to the frequency of the

Table 1 Characteristics of controls and patients with sleep disordered breathing (SDB)

\begin{tabular}{|c|c|c|c|c|c|c|c|}
\hline & $\begin{array}{l}\text { Controls } \\
(n=17)\end{array}$ & $\begin{array}{l}\text { All patients } \\
(\mathrm{n}=50)\end{array}$ & p valuet & $\begin{array}{l}\text { Mild group } \\
(n=5)\end{array}$ & $\begin{array}{l}\text { Moderate group } \\
(n=19)\end{array}$ & $\begin{array}{l}\text { Severe group } \\
(n=26)\end{array}$ & p valuef \\
\hline \multicolumn{8}{|l|}{ Characteristics } \\
\hline Age (years) & $42.1(7.82)$ & $48.5(12.7)$ & 0.0378 & $31.4(8.82)$ * & $50.1(8.27)$ * & $50.6(13.8)$ * & 0.0020 \\
\hline BMI $\left(\mathrm{kg} / \mathrm{m}^{2}\right)$ & $24.3(2.57)$ & $28.5(4.54)$ & 0.0004 & $25.6(3.29)$ & $27.4(3.39)$ ** & $29.9(5.08)$ *** & 0.0003 \\
\hline Sleepiness Epworth score (0-24) & $4.76(3.29)$ & $11.0(4.37)$ & 0.0001 & $13.6(4.34)$ ** & $10.9(3.45)^{* * *}$ & $10.5(4.89)$ *** & 0.0001 \\
\hline Gag reflex intensity (0-3) & $1.62(0.52)$ & $1.54(0.82)$ & 0.6809 & $2.10(0.89)$ & $1.50(0.81)$ & $1.46(0.80)$ & 0.4917 \\
\hline Tobacco consumption (packs/year) & $13.1(14.7)$ & $13.5(17.0)$ & 0.9193 & $6.15(7.94)$ & $11.4(11.8)$ & $16.4(20.6)$ & 0.8327 \\
\hline Alcohol consumption (drinks/day) & $0.70(0.74)$ & $1.88(2.04)$ & 0.0377 & $0.51(0.47)$ & $1.12(1.22)$ & $2.70(2.36)$ ** & 0.0079 \\
\hline Spicy food consumption (0-3) & $0.79(0.95)$ & $1.09(0.93)$ & 0.2146 & $0.60(0.55)$ & $0.76(1.03)$ & $1.44(0.79)$ * & 0.0161 \\
\hline \multicolumn{8}{|l|}{ Sleep study } \\
\hline Mean nocturnal $\mathrm{SaO}_{2}(\%)$ & $95.3(1.16)$ & $92.7(2.55)$ & 0.0001 & $95.5(1.95)$ & $93.8(2.27)$ * & $91.4(2.01) * * *$ & 0.0001 \\
\hline Minimal nocturnal $\mathrm{SaO}_{2}(\%)$ & $91.8(1.89)$ & $79.5(11.2)$ & 0.0001 & $91.6(1.82)$ & $83.6(9.07)^{* * *}$ & $74.4(10.8)^{* * *}$ & 0.0001 \\
\hline $\begin{array}{l}\text { Percentage of time spent below } 90 \% \text { of } \\
\mathrm{SaO}_{2}(\%)\end{array}$ & \multicolumn{6}{|c|}{ Percentage of time spent below $90 \%$ of } & 0.0001 \\
\hline RDI (events/h of sleep) & NA & $47.0(20.5)$ & NA & $28.7(10.7)$ & $41.4(16.2)$ & $54.6(21.5)$ & 0.0172 \\
\hline AHI (events/h of sleep) & NA & $41.7(21.3)$ & NA & $15.6(5.74)$ & 32.5 (11.9) & $53.4(21.0)$ & 0.0001 \\
\hline Apnoea index (events/h of sleep) & NA & $9.54(13.1)$ & NA & $0.25(0.22)$ & $6.54(9.05)$ & $13.5(15.5)$ & 0.0026 \\
\hline Hypopnoea index (events/h of sleep) & NA & $32.2(16.7)$ & NA & $15.3(5.59)$ & $26.0(9.37)$ & $39.9(18.2)$ & 0.0004 \\
\hline Flow limitation index (events/h of sleep) & NA & $5.31(6.48)$ & NA & $13.2(5.18)$ & $8.90(6.89)$ & $1.18(1.97)$ & 0.0001 \\
\hline Obstructive index (events/h of sleep) & NA & $45.3(19.8)$ & NA & $26.7(10.6)$ & $40.1(15.9)$ & $52.6(20.4)$ & 0.0128 \\
\hline Central index (events/h of sleep) & NA & $1.75(2.51)$ & NA & $2.07(1.36)$ & $1.37(2.19)$ & $1.96(2.90)$ & 0.5527 \\
\hline \multicolumn{8}{|l|}{ Pharyngeal sensitivity } \\
\hline Disappearance threshold at baseline (l/min) & $0.40(0.19)$ & $0.85(0.40)$ & 0.0001 & $0.70(0.29)$ * & $0.77(0.36)$ ** & $0.94(0.43) * * *$ & 0.0005 \\
\hline $\begin{array}{l}\text { Disappearance threshold after } 3 \\
\text { ancesthesias (I/min) }\end{array}$ & $1.10(0.30)$ & $2.47(1.85)$ & 0.0002 & $1.59(0.60)$ & $2.23(1.78)$ * & $2.85(2.04) * * *$ & 0.0013 \\
\hline $\begin{array}{l}\text { Disappearance threshold slope (arbitrary } \\
\text { unit) }\end{array}$ & $0.22(0.09)$ & $0.54(0.58)$ & 0.0778 & $0.28(0.15)$ & $0.46(0.55)$ & $0.66(0.64)$ & 0.2665 \\
\hline Appearance threshold at baseline $(1 / \mathrm{min})$ & $0.26(0.06)$ & $0.62(0.44)$ & 0.0003 & $0.28(0.06)$ & $0.63(0.48)^{* * *}$ & $0.67(0.44) * * *$ & 0.0013 \\
\hline $\begin{array}{l}\text { Appearance threshold after } 3 \text { anaesthesias } \\
(1 / \mathrm{min})\end{array}$ & $0.93(0.25)$ & $2.33(2.03)$ & 0.0004 & $1.05(0.15)$ & $1.95(1.62)$ ** & $2.86(2.33) * \star *$ & 0.0010 \\
\hline Appearance threshold slope (arbitrary unit) & $0.22(0.07)$ & $0.56(0.57)$ & 0.0074 & $0.25(0.05)$ & $0.42(0.44)$ & $0.72(0.66)$ ** & 0.0100 \\
\hline
\end{tabular}

$\mathrm{BMI}$, body mass index; $\mathrm{SaO}_{2}$, oxygen saturation; $\mathrm{RDI}$, respiratory disturbance index; $\mathrm{AHI}$, apnoea-hypopnoea index.

Patients were classified into three groups according to the proportion of the different respiratory events constituting the sleep disordered breathing: "mild" patients had apnoeahypopnoea events <60\%; "moderate" patients had apnoea-hypopnoea events 60-90\%; "severe" patients had apnoea-hypopnoea events $>90 \%$.

†Comparisons between controls and all patients using Mann-Whitney $U$ test.

†Analysis of variance (Kruskal-Wallis test) between controls and the three groups of patients, or only between the three groups of patients when data in controls were not available.

Each group of patients was compared with controls using the Mann-Whitney $U$ test: ${ }^{*} p<0.05,{ }^{* *} p<0.01,{ }^{* * *} p<0.001$.

Gag reflex was scored 0 (absent), 1 (decreased), 2 (normal) and 3 (exaggerated). Spicy food consumption was scored 0 (no consumption), 1 (little), 2 (moderate) and 3 (high consumption). Obstructive index includes mixed plus obstructive apnoeas and hypopnoeas and flow limitation episodes. Central index includes central apnoeas and hypopnoeas. 
respiratory events without prejudging their respective proportion, we also defined SDB severity according to the proportion of the different respiratory events constituting the SDB. Indeed, as SDB pathophysiologically corresponds to a continuous spectrum of overlapping entities with increasing severity related to collapsibility of the upper airway (from flow limitation events to obstructive hypopnoeas and apnoeas), patients were classified into three groups according to the proportion of the different respiratory events. The cutoff values were arbitrarily chosen and allowed us to distinguish between patients with a high proportion of inspiratory flow limitations, patients with a high proportion of apnoea-hypopnoea, and patients in an intermediate situation. The less severely affected group ("mild") included patients with apnoea-hypopnoea events $<60 \%$ and flow limitation episodes $>40 \%$ of events; the moderately affected group ("moderate") included patients with apnoea-hypopnoea events of $60-90 \%$ and flow limitation episodes of $10-$ $40 \%$ of events; and the most severely affected group ("severe") included patients with apnoea-hypopnoea events $>90 \%$ and flow limitation episodes $<10 \%$ of events. The differences between these three subgroups of patients also referred to the severity of the AHI, RDI, and oxygen desaturation as reflected by mean and minimum nocturnal $\mathrm{SaO}_{2}$ (table 1). The significance was set at $\mathrm{p}<0.05$.

Finally we assessed the sensitivity of the test (that is, how effective it is at identifying patients with SDB) in the same set of patients. For these calculations the pharyngeal sensitivity of the patients was considered abnormal when at least one parameter of the test (appearance or disappearance thresholds or slopes) was higher than the 97.5th percentile of values obtained in the 17 control subjects. The accuracy of the test (percentage of SDB correctly diagnosed (RDI $>20 /$ hour) for all patients and for each subgroup of patients) was determined by comparison with the gold standard polygraphy.

\section{RESULTS}

Pharyngeal sensitivity was easily measured in all subjects, regardless of gag reflex intensity or mouth opening. The characteristics of the patients are summarised in table 1. Patients were older and had a higher BMI, sleepiness, and alcohol consumption than controls.

Differences between the three subgroups of patients and controls were then assessed. The "mild" group was younger while the two other groups were older with a higher BMI. All three groups had more sleepiness than controls. Nocturnal desaturation was greater in the "moderate" and "severe" groups, while controls and the "mild" group were not different. Alcohol and spicy food consumption was higher in the "severe" group while gag reflex intensity did not differ between the three groups. Gag reflex was re-assessed in some patients at the end of the procedure and no change in intensity was found despite the anaesthesia.

\section{Pharyngeal sensitivity in controls and repeatability of the test}

While no significant interaction was found between measurement conditions and sessions for both the disappearance $(\mathrm{p}=0.793)$ and appearance $(\mathrm{p}=0.357)$ procedures, the two way ANOVA revealed a significant difference between the four measurement conditions (baseline and successive anaesthesias) of each session for both procedures $(p=0.0001)$. The sensory thresholds significantly increased from the first anaesthesia (post hoc Scheffé test). In contrast, the increase in sensory threshold following the second and the third anaesthesias was weaker (no significant difference between successive anaesthesias) leading to a plateau effect (fig 2). This evolution was repeatable with no significant difference being found between the two sessions (disappearance threshold: $\mathrm{p}=0.537$; appearance threshold: $\mathrm{p}=0.346$ ).

As shown by the Bland-Altman plots in the lower panel of fig 2 and table 2, the differences between the two sessions were close to zero indicating a small bias over time for both the disappearance and appearance threshold procedures. At least $90 \%$ of measurements were within the limits of agreement. These limits were, however, wider for the disappearance thresholds than for the appearance thresholds. The calculation of the repeatability coefficient led to similar results whatever the statistical analysis (table 2). The smaller the repeatability coefficient, the more repeatable the measurements. As shown by the grouping of values and the narrow limits of agreement (fig 2), smaller repeatability coefficients were obtained at baseline, particularly for the appearance threshold procedure. Conversely, the higher the intraclass correlation coefficient, the more repeatable the measurements. However, because calculation of the intraclass correlation coefficient depends on the variance in the population, the smaller the variance between subjects the smaller the intraclass correlation coefficient, despite a high repeatability as shown by the repeatability coefficient (table 2). Indeed, as shown by $\mathrm{F}$ ratios for subjects and $\mathrm{p}$ values, while no difference between subjects was noted at baseline appearance threshold, a significant difference occurred between subjects under anaesthesia suggesting a heterogeneous response to anaesthesia (that is, individual susceptibility). For the disappearance procedure, a trend for a difference between subjects was noted from baseline. Conversely, as shown by $\mathrm{F}$ ratio and $\mathrm{p}$ values for repeated measures, subjects behaved similarly between the two sessions.

\section{Pharyngeal sensitivity in controls and patients}

Patients had a higher baseline pharyngeal sensory detection threshold. Differences between controls and patients were enhanced by anaesthesia, as reflected by the slopes (table 1 and fig 3).

Sensory thresholds were significantly correlated with age (appearance threshold at baseline $(r=0.33, \mathrm{p}=0.024)$ and after anaesthesia (first anaesthesia: $r=0.48, \mathrm{p}=0.001$; second anaesthesia: $r=0.37, \mathrm{p}=0.010$; third anaesthesia: $r=0.35, \mathrm{p}=0.017)$. They were positively correlated with BMI for both the disappearance threshold (baseline: $r=0.33$, $\mathrm{p}=0.027$; first anaesthesia: $r=0.38, \mathrm{p}=0.010$ ) and the appearance threshold (first anaesthesia: $r=0.29, \mathrm{p}=0.049$ ). For the other sensory thresholds a trend emerged that did not reach statistical significance.

\section{Pharyngeal sensitivity and severity of SDB}

Individual values for baseline sensory thresholds and slopes in controls and the three groups of patients are shown in fig 4 . At baseline, control values were grouped in a narrow range, particularly for the appearance sensory threshold, while values in "moderate" and "severe" patients were widely dispersed with a mean value significantly higher than that of controls. In contrast, pharyngeal sensory perception of the "mild" group was close to that of controls (see also table 1 and fig 3). However, some patients in the two most severe groups had sensory thresholds similar to controls. Patients with normal sensitivity were compared with patients exhibiting an impaired sensitivity in the same groups. Patients with normal sensitivity had a higher proportion of flow limitation episodes (15.0 (9.32) $v 9.07$ (11.4)\%, $\mathrm{p}=$ $0.049)$ reflecting less severe SDB, and a lower BMI (25.4 (4.24) $v 29.6(4.34), \mathrm{p}=0.014)$ than patients with impaired pharyngeal sensitivity.

Significant differences in response to topical anaesthesia were identified in the different groups (fig 4, lower panel). 

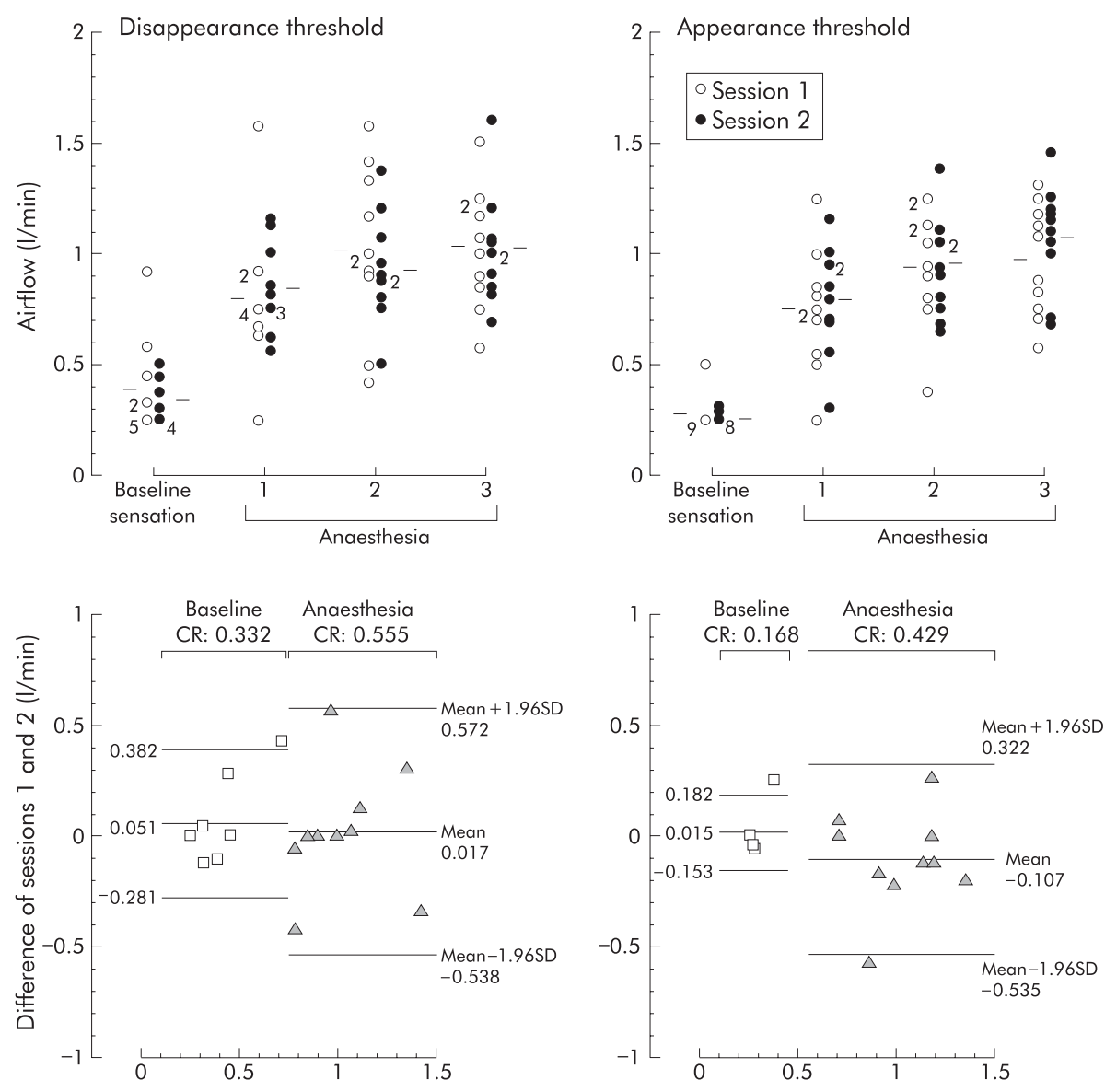

Average of sessions 1 and $2(1 / \mathrm{min})$

Average of sessions 1 and $2(1 / \mathrm{min})$

Figure 2 Repeatability of the procedure. Ten control subjects were re-evaluated after a mean (SD) interval of 31.9 (3) weeks before and after three successive administrations of topical anaesthetic. Upper panel: Individual circles correspond to the measurement of one subject. Numbered circles indicate the number of subjects with a similar value. Mean values are indicated by the horizontal bars. Lower panel: Bland-Altman plot where the differences between the repeated measurements are plotted against their average, with $95 \%$ limits of agreement (mean $+1.96 S D$ ). SD is the standard deviation of the differences between paired measurements. Measurement values obtained at baseline (squares) and after the last anaesthesia (triangles) are shown; the last anaesthesia was chosen as an example of the effect of anaesthesia on the procedure repeatability. CR is the British Standards Institution repeatability coefficient calculated as 1.96 times the standard deviation of the differences.

While controls and "mild" patients behaved similarly, some patients in the "moderate" and "severe" groups clearly had an increased response to anaesthesia. This allowed us to discriminate some patients with normal baseline values from controls, and moderate from severely affected groups, while their baseline values were close (fig 3).

Sensory detection thresholds were negatively correlated with the flow limitation index (disappearance threshold at baseline and after anaesthesia: $r=-0.32, \quad \mathrm{p}=0.030$; $r=-0.30, \mathrm{p}=0.050$ respectively). In contrast, detection thresholds were positively correlated with the obstructive hypopnoea index (appearance threshold at baseline $(r=0.28$, $\mathrm{p}=0.058$ ) and after anaesthesia (first anaesthesia: $r=0.33$, $\mathrm{p}=0.026$; second anaesthesia: $r=0.32, \mathrm{p}=0.029$; third anaesthesia: $r=0.29, \mathrm{p}=0.044)$; disappearance threshold after one anaesthesia: $r=0.30, \mathrm{p}=0.038$ ). While a trend was apparent, no significance was reached for the other disappearance sensory thresholds.

The higher the percentage of hypopnoeas constituting SDB, the higher the sensory threshold (disappeareance sensory threshold after anaesthesia (first anaesthesia: $r=0.29$, $\mathrm{p}=0.049$; second anaesthesia: $r=0.33, \mathrm{p}=0.030$ ); appearance sensory threshold at baseline $(r=0.25, \mathrm{p}=0.085)$ and after anaesthesia (first anaesthesia: $r=0.28, \mathrm{p}=0.055$; second anaesthesia: $r=0.32, \mathrm{p}=0.028$; third anaesthesia: $r=0.37, \mathrm{p}=0.011)$ ). Similarly, the percentage of apnoeas + hypopnoeas constituting SDB was positively correlated with the sensory thresholds (disappearance sensory detection thresholds at baseline $(r=0.32, \mathrm{p}=0.032)$ and after anaesthesia $(r=0.30, \mathrm{p}=0.053))$. In contrast, there was no significant correlation or any trend between sensory detection thresholds and the classical AHI and RDI and nocturnal oxygen saturation.

\section{Pharyngeal sensitivity and diagnosis of SDB}

Overall, the sensitivity of the test for SDB diagnosis (RDI $>20$ /hour) was $79.6 \%$ and decreased from the most severe $(88.5 \%)$ to the least severe $(50 \%)$ group, with an intermediate sensitivity $(73.7 \%)$ for the "moderate" group.

\section{DISCUSSION}

The simple new approach described in this study enabled us to measure pharyngeal sensory perception easily and reliably without any side effects. Using this system, we have confirmed that impairment of pharyngeal sensory perception is correlated with the severity of SDB. When evaluated as a diagnostic tool, the test showed a high repeatability and sensitivity for SDB diagnosis in our sleep clinic population. 


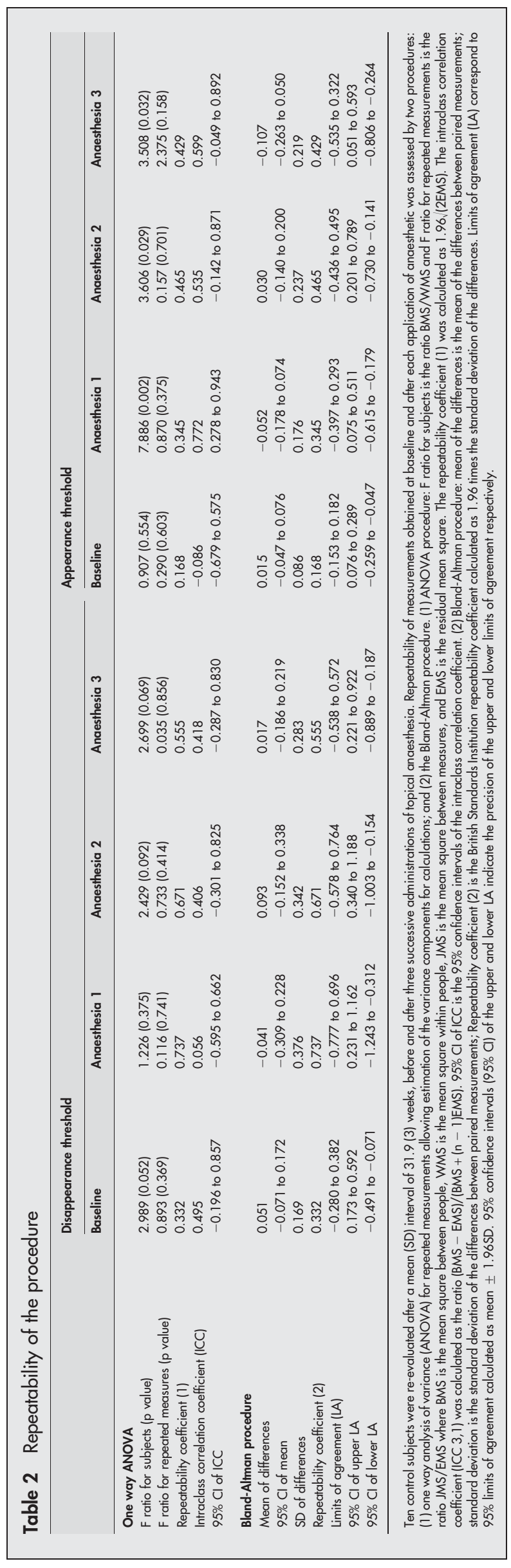

Pharyngeal sensory impairment: a factor explaining increased pharyngeal collapsibility in SDB

The pharynx functions as a collapsible conduit which tends to collapse during negative pressure induced by inspiration. ${ }^{1}$ Factors predisposing to upper airway collapse include anatomical narrowing and/or abnormal collapsibility of the upper airway. ${ }^{15}$ Patients with obstructive sleep apnoea have an anatomically small upper airway, even during wakefulness, which may be the result of obesity through fat deposition around the upper airway, ${ }^{16}$ small mandible (micrognathia), jaw position (retrognathia), soft palate elongation and thickness, tonsil or tongue hypertrophy. Pharyngeal patency is also largely dependent on the activity of PDM through a crucial protective reflex activated by inspiratory negative pressure. ${ }^{5}{ }^{17}$ In subjects with sleep apnoea this reflex functions during wakefulness as a compensatory mechanism, increasing dilator muscle activity in response to anatomical narrowing (neuromuscular compensation). ${ }^{18}$ During sleep this neuromuscular compensation is lost, leading to partial or complete collapse of the upper airway. ${ }^{19}$ The impairment of reflex dilation of the upper airway can occur at two levels: the afferent limb (pharyngeal sensitivity including receptors and sensory nerves) and the efferent limb (motor nerves and muscles). The purpose of our study was to investigate the afferent limb without prejudging the aetiology of the sensory impairment.

The mechanisms underlying the impairment of pharyngeal sensitivity may include chronic upper airway inflammation with mucosal oedema, fat deposition, and pharyngeal neuropathy. The presence of oedema has been histologically demonstrated..$^{20}$ Oedema may be related, at least in part, to repeated mechanical trauma in the upper airway from snoring-related vibration and apnoea-related suction and stretching. ${ }^{22}$ Such oedema is indeed reduced during chronic treatment with continuous positive airway pressure. ${ }^{23}$ Infectious disease, allergy, tobacco, alcohol, gastro-oesophageal reflux, oral hygiene, and hot spicy foods may also contribute to upper airway inflammation as suggested by the higher intake of alcohol and spicy food in our apnoeic group. However, the higher rate of spicy food consumption observed may have been a consequence rather than a cause of decreased pharyngeal sensitivity.

The existence of pharyngeal neuropathy in patients with SDB is supported by the increased density in sensory nerve endings in biopsy specimens from the soft palate mucosa of snorers and apnoeic subjects, ${ }^{24}$ and by focal degeneration of the myelin sheaths and axons in uvulopalatopharyngoplasty specimens from subjects with apnoea. ${ }^{20}$ These observations suggest that progression from mild occasional snoring to heavy habitual snoring and then to sleep apnoea may represent a progressive local neuropathy. ${ }^{22}$ Mechanisms that could lead to sensory receptor or nerve damage in the upper airway may include mucosal oedema resulting from mechanical stress, vascular changes ${ }^{25}$ and inflammation that could interfere with the function of nerve endings, and direct vibration-related injury analogous to nerve lesions in the upper extremities of hand held vibrating tool users. ${ }^{26}$ In addition, the course of this vibration syndrome may be affected by associated diseases, ${ }^{27}$ smoking, neurotoxic drugs, and alcohol intake. As we have previously shown for peripheral nerves, ${ }^{28}$ hypoxia related to apnoea may also contribute to the neuropathy. All these mechanisms may explain the difference in sensitivity impairment observed between the three groups of patients. The "severe" group was overweight and had the most severe respiratory events which are stressful for the upper airway and lead to significant oxygen desaturation. In addition, this group was older, suggesting a longer disease duration and possibly a physiological age-related decreased sensitivity, ${ }^{29}$ as for the 

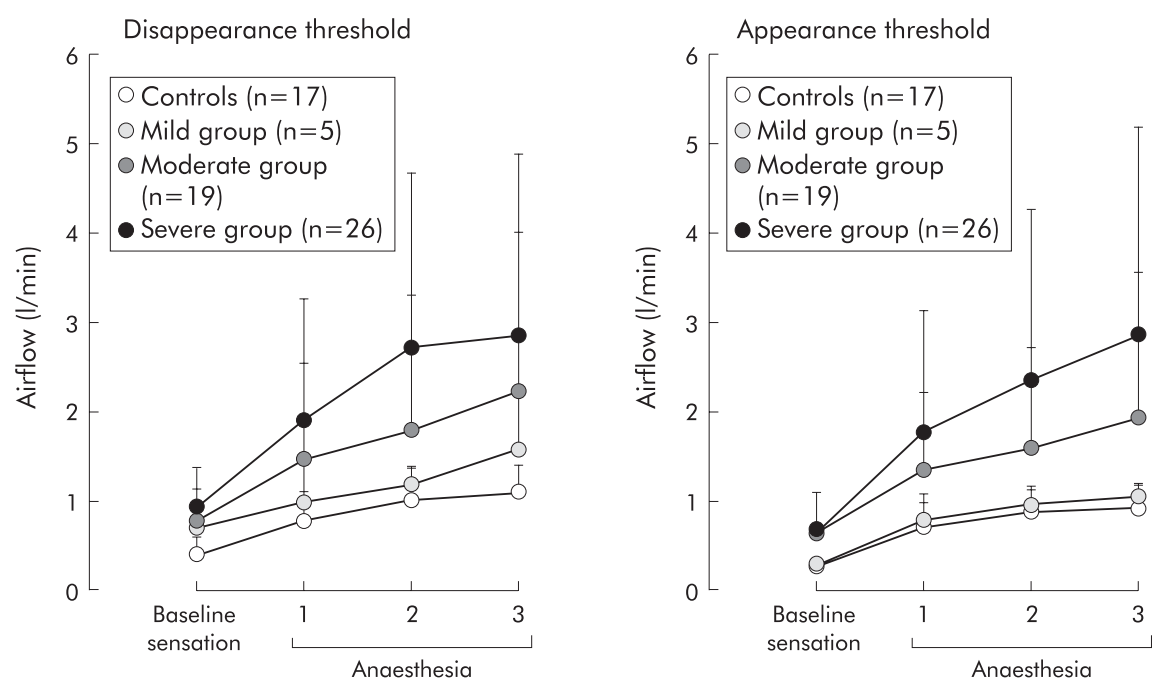

Figure 3 Pharyngeal sensory thresholds in controls and in the three patients groups before and after anaesthesia. The patients were classified into three groups according to the proportion of the different respiratory events constituting the sleep disordered breathing: "mild" patients had apnoeahypopnoea events <60\%; "moderate" patients had apnoea-hypopnoea events 60-90\%; "severe" patients had apnoea-hypopnoea events $>90 \%$. Left panel: Disappearance sensory perception threshold. Right panel: Appearance sensory perception threshold. Values are mean (SD).

peripheral nerves. Such difference in sensitivity impairment between the three groups may in turn contribute to the difference in SDB severity.

\section{Advantages and limitations of the procedure for measuring pharyngeal sensitivity}

Our device allowed easy and non-invasive assessment of pharyngeal sensitivity in terms of tactile perception without requiring specialised materials or professional skills. The device is simple and the procedure can be performed with standard equipment readily available in any sleep laboratory.

Compared with previously described procedures, ${ }^{13}{ }^{30} 31$ we were able to assess pharyngeal sensitivity in subjects with an intense gag reflex and small oropharyngeal cavity, thus excluding possible bias selection. Using devices of different calibre, our system could be adapted to various degrees of mouth opening. As previously described, ${ }^{32}$ unlike measurement of pharyngeal sensation, gag reflex was not informative since we found no difference between groups.

Measurements were obtained rapidly (around 30 minutes) and were repeatable, thus making the test reliable and unconstrained for both the patient and the investigator. One limitation of psychophysical evaluations is the subjective character of the answers which rely on the subject's cooperation. However, although our results require further validation in a larger population, the repeatability of the measurements (fig 2, table 2) shows that the subjectivity of the answers was reduced by repeating the measurements during each test session and by using random null stimuli.

The anatomical region tested was the soft palate because of its critical involvement in the pathophysiology of SDB (see above). Experiments are currently in progress in our laboratory to test additional areas. Indeed, the adjustability of the guide allows testing of other oropharyngeal areas such as the tonsil pillar, hard palate and uvula (data not shown) which are either differently innervated and/or differently exposed to mechanical stress during sleep.

We have shown that pharyngeal sensitivity is differentially impaired according to the severity of SDB in terms of the type of respiratory events while no significant correlation was found with the classical AHI and RDI or with nocturnal desaturation. This argues for the pathophysiological involvement of pharyngeal sensitivity in collapsibility of the upper airway. Compared with patients in the two most severely affected groups, patients in the "mild" group were younger and suffer from SDB with a high proportion of flow limitation episodes and no nocturnal desaturation. The "mild" group therefore represented patients suffering from upper airway resistance syndrome or mild obstructive sleep apnoea, while the "moderate" and "severe" groups represented hypopnoeic and apnoeic patients respectively. Patients in the "mild" group had pharyngeal sensitivity which was close to controls or intermediate between controls and "moderate/severe" patients. These results are in agreement with a previous study showing that collapsibility of the upper airway during sleep in upper airway resistance syndrome is intermediate between that of normal subjects and patients with mild to moderate obstructive sleep apnoea. ${ }^{33}$

Our ability to demonstrate a difference in impairment of pharyngeal sensitivity testifies to the higher capacity of discrimination of our procedure. Indeed, our stimulus consisted of an air pulse administered at a constant distance from the mucosa. Unlike previous studies, ${ }^{13}{ }^{30}{ }^{31}$ we did not use any device that may induce gag reflex or interfere with the sensory perception because of the difficulty in maintaining a constant contact pressure with the mucosa, particularly on the soft palate. Another explanation for the capacity to discriminate between SDB severity levels was the use of topical anaesthesia. As we suspected, it clearly enabled us to separate some patients with normal baseline values from controls, and medium from severely affected groups while values were close at baseline. However, despite a dose-effect response, differences between subgroups were not statistically significant due to overlapping values. Indeed, even patients with severe SDB could have normal pharyngeal sensitivity at baseline and under anaesthesia (see below). Compared with previous studies, ${ }^{89}$ the anaesthesia was light and localised since the gag reflex was unchanged by the procedure. Such anaesthesia was, however, sufficient to sensitise the test from the first spray and may be useful to simplify the procedure by decreasing the number of measurements and the duration of the examination.

\section{Pharyngeal sensitivity: a tool to predict the severity of SDB?}

Overall, this test (appearance and disappearance thresholds, slopes) revealed a high sensitivity for identifying patients suffering from sleep apnoea syndrome in our sleep clinic 

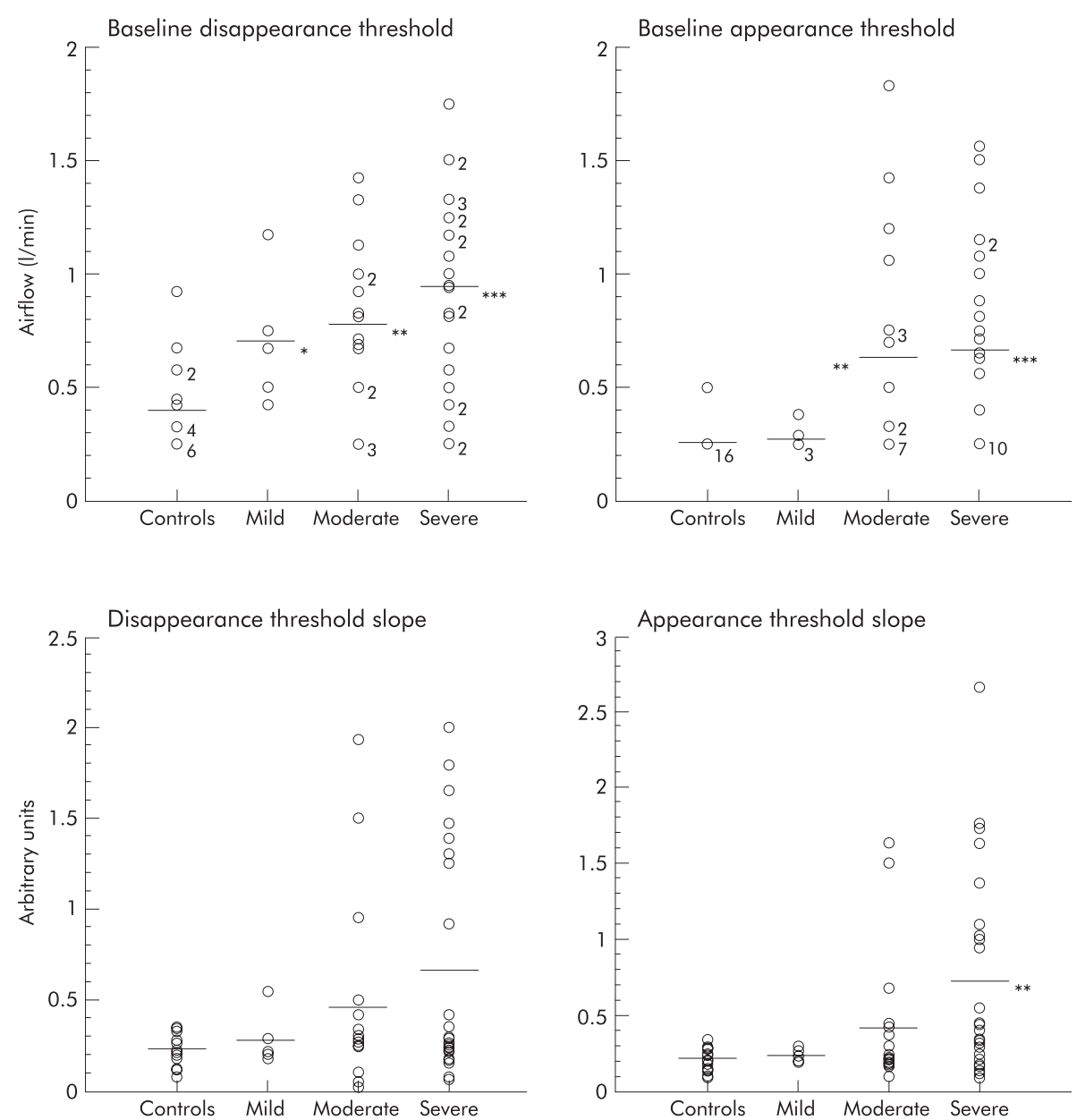

Figure 4 Baseline sensory threshold and slope values for individual subjects in the control group and the three subgroups of patients. Left panel: baseline (upper) and slope (lower) values for the disappearance threshold; right panel: baseline (upper) and slope (lower) values for the appearance threshold. Individual circles correspond to the measurement of one subject. Circles with numbers indicate the number of subjects with similar values. Mean values are indicated by the horizontal bars. ${ }^{*} p<0.05,{ }^{* *} p<0.01,{ }^{* * *} p<0.001 v$ controls. Note the similar grouping of values in controls compared with the higher and widely dispersed values in patients suffering from increasingly severe SDB. Some patients, even in the most severe groups, had similar sensory thresholds and slopes than controls.

population. With the chosen cut-off value, pharyngeal sensitivity considered abnormal was systematically associated with SDB. In contrast, the existence of SDB was not systematically associated with an impairment of pharyngeal sensitivity, as shown by the sensitivity of the test in the "mild" (50\%), "moderate" (73.7\%), and "severe" (88.5\%) groups. These findings confirm that pharyngeal sensitivity is only one determinant-together with airway anatomyamong the predisposing factors of collapse of the upper airway. In subjects with an anatomical predisposition to upper airway obstruction, partial impairment of the upper airway dilating muscle function may be sufficient to cause collapse of the airway. On the other hand, patients with a normal upper airway anatomy may be less vulnerable to impairment of the reflex dilation of the airway. Both the existence and severity of SDB are not dependent only on impairment of pharyngeal sensitivity. Thus, patients with similar pharyngeal sensitivity may exhibit different upper airway collapsibility since the motor part (efferent fibres and muscles) of the dilating reflex, ${ }^{34}$ not explored by our procedure, and the pharyngeal anatomy may also contribute to impairment of upper airway dilation.

Collapsibility of the upper airway and resulting SDB therefore appear to result from an equation including several factors, each of them being weighed by different coefficients that may be characteristic for an individual and may also evolve over time and with treatment. Among these factors, the most severely affected group in our study had a higher BMI and a higher alcohol intake which represent additional anatomical and functional factors that are likely to worsen SDB. In the multifactorial equation resulting in SDB, pharyngeal sensitivity is probably one of the key factors in our study, predictive not only of the existence but also of the severity of SDB.

Taken together, our results suggest a flow diagram for the diagnosis of sleep apnoea: on the one hand, lean and young patients preferentially suffer from upper airway resistance events and/or mild hypopnoeas. The pharyngeal sensitivity is then normal or subnormal. In this context, full polysomnography including respiratory effort assessment is required for diagnosis. ${ }^{14}$ On the other hand, more obese or older patients preferentially suffer from hypopnoea and/or apnoea. Impairment of pharyngeal sensitivity in such patients could provide a simplified diagnostic procedure. This proposed diagnostic flowchart should be prospectively validated in a larger sleep clinic population as well as in the general population.

In conclusion, we have developed a simple, repeatable, and safe procedure which confirms the presence of impaired pharyngeal sensitivity in patients with SDB and have shown 
that such impairment is correlated with the severity of SDB Although this new procedure needs to be fully validated in a larger population, its simplicity suggests that it may be of use in routine clinical practice to evaluate the role of pharyngeal sensitivity in the pathophysiology of SDB and its value for simplification of the SDB diagnosis procedure.

\section{ACKNOWLEDGEMENTS}

The authors thank Drs B Lepaulle and F Arbib for their contribution to patient recruitment, C Loiodice and M Selek for polysomnographic data collection, and D Villemain, J L Quesada and J L Bosson for statistical advice.

\section{Authors' affiliations}

M Dematteis, P Lévy, J-L Pépin, Laboratoire du sommeil et Laboratoire HP2 (INSERM ESPRI EA3745), Centre Hospitalier Universitaire, BP 217 38043 Grenoble Cedex 09, France

Supported by a grant from ANTADIR (Association fédérative Nationale pour le Traitement à Domicile de l'Insuffisance Respiratoire chronique).

\section{REFERENCES}

1 Deegan PC, McNicholas WT. Pathophysiology of obstructive sleep apnoea. Eur Respir J 1995:8:1161-78.

2 Hudgel DW. Variable site of airway narrowing among obstructive sleep apnea patients. J Appl Physiol 1986;61:1403-9.

3 Wiegand DA, Latz B, Zwillich CW, et al. Upper airway resistance and geniohyoid muscle activity in normal men during wakefulness and sleep. J Appl Physiol 1990;69:1252-61.

4 Tangel DJ, Mezzanotte WS, Sandberg EJ, et al. Influences of NREM sleep on the activity of tonic vs. inspiratory phasic muscles in normal men. J Appl Physiol 1992; 73:1058-66.

5 Horner RL, Innes JA, Murphy K, et al. Evidence for reflex upper airway dilator muscle activation by sudden negative airway pressure in man. J Physiol 1991;436:15-29.

6 Horner RL, Innes JA, Holden HB, et al. Afferent pathway(s) for pharyngeal dilator reflex to negative pressure in man: a study using upper airway anaesthesia. J Physiol 1991;436:31-44.

7 DeWeese EL, Sullivan TY. Effects of upper airway anesthesia on pharyngea patency during sleep. J Appl Physiol 1988;64:1346-53.

8 McNicholas WT, Coffey M, McDonnell T, et al. Upper airway obstruction during sleep in normal subjects after selective topical oropharyngeal anesthesia. Am Rev Respir Dis 1987;135:1316-9.

9 Chadwick GA, Crowley P, Fitzgerald MX, et al. Obstructive sleep apnea following topical oropharyngeal anesthesia in loud snorers. Am Rev Respir Dis 1991:143(4 P+1):810-3.

10 Berry RB, Kouchi KG, Bower JL, et al. Effect of upper airway anesthesia on obstructive sleep apnea. Am J Respir Crit Care Med 1995;151:1857-61.

11 Young T, Palta M, Dempsey J, et al. The occurrence of sleep-disordered breathing among middle-aged adults. N Engl J Med 1993:328:1230-5.

12 Mayer P, Pepin JL, Bettega G, et al. Relationship between body mass index, age and upper airway measurements in snorers and sleep apnoea patients. Eur Respir J 1996;9:1801-9.
13 Kimoff RJ, Sforza E, Champagne $\mathrm{V}$, et al. Upper airway sensation in snoring and obstructive sleep apnea. Am J Respir Crit Care Med 2001;164:250-5.

14 American Academy of Sleep Medicine Task Force. Sleep-related breathing disorders in adults: recommendations for syndrome definition and measurement techniques in clinical research. Sleep 1999:22:667-89.

15 Gleadhill IC, Schwartz AR, Schubert N, et al. Upper airway collapsibility in snorers and in patients with obstructive hypopnea and apnea. Am Rev Respir Dis 1991; 143:1300-3.

16 Horner RL, Mohiaddin RH, Lowell DG, et al. Sites and sizes of fat deposits around the pharynx in obese patients with obstructive sleep apnoea and weight matched controls. Eur Respir J 1989;2:613-22.

17 Mathew OP, Abu-Osba YK, Thach BT. Influence of upper airway pressure changes on genioglossus muscle respiratory activity. J Appl Physiol 1982:52:438-44.

18 Mezzanotte WS, Tangel DJ, White DP. Waking genioglossal electromyogram in sleep apnea patients versus normal controls (a neuromuscular compensatory mechanism). J Clin Invest 1992;89:1571-9.

19 Wheatley JR, Mezzanotte WS, Tangel DJ, et al. Influence of sleep on genioglossus muscle activation by negative pressure in normal men. Am Rev Respir Dis 1993;148:597-605.

20 Woodson BT, Garancis JC, Toohill RJ. Histopathologic changes in snoring and obstructive sleep apnea syndrome. Laryngoscope 1991;101(12 Pt 1):1318-22

21 Sekosan M, Zakkar M, Wenig BL, et al. Inflammation in the uvula mucosa of patients with obstructive sleep apnea. Laryngoscope 1996;106:1018-20.

22 Friberg D. Heavy snorer's disease: a progressive local neuropathy. Acta Otolaryngol 1999; 11:925-33

23 Ryan CF, Lowe AA, Li D, et al. Magnetic resonance imaging of the upper airway in obstructive sleep apnea before and after chronic nasal continuous positive airway pressure therapy. Am Rev Respir Dis 1991;144:939-44.

24 Friberg D, Gazelius B, Hokfelt T, et al. Abnormal afferent nerve endings in the soft palatal mucosa of sleep apnoics and habitual snorers. Regul Pept 1997:71:29-36.

25 Friberg D, Gazelius B, Lindblad LE, et al. Habitual snorers and sleep apnoics have abnormal vascular reactions of the soft palatal mucosa on afferent nerve stimulation. Laryngoscope 1998;108:431-6.

26 Takeuchi T, Futatsuka $M$, Imanishi $H$, et al. Pathological changes observed in the finger biopsy of patients with vibration-induced white finger. Scand J Work Environ Health 1986;12(4 Spec No):280-3.

27 Dematteis M, Pépin JL, Jeanmart $M$, et al. Charcot-Marie-Tooth disease (CMTIA) and sleep apnoea syndrome: a family study. Lancet 2001;357:267-72.

28 Mayer P, Dematteis M, Pépin JL, et al. Peripheral neuropathy in sleep apnea. A tissue marker of the severity of nocturnal desaturation. Am J Respir Crit Care Med 1999; 159:213-9.

29 Aviv JE, Martin JH, Jones ME, et al. Age-related changes in pharyngeal and supraglottic sensation. Ann Otol Rhinol Laryngol 1994;103:749-52.

30 Larsson H, Carlsson-Nordlander B, Lindblad LE, et al. Temperature thresholds in the oropharynx of patients with obstructive sleep apnea syndrome. Am Rev Respir Dis 1992;146(5 Pt 1):1246-9.

31 Guilleminault $\mathrm{C}$, Li K, Chen NH, et al. Two-point palatal discrimination in patients with upper airway resistance syndrome, obstructive sleep apnea syndrome, and normal control subjects. Chest 2002;122:866-70.

32 Davies AE, Kidd D, Stone SP, et al. Pharyngeal sensation and gag reflex in healthy subjects. Lancet 1995;345:487-8.

33 Gold AR, Marcus CL, Dipalo F, et al. Upper airway collapsibility during sleep in upper airway resistance syndrome. Chest 2002;121:1531-40.

34 Friberg D, Ansved T, Borg K, et al. Histological indications of a progressive snorers disease in an upper airway muscle. Am J Respir Crit Care Med $1998 ; 157: 586-93$ 